\title{
The Kumaraswamy Marshall-Olkin Log-Logistic Distribution with Application
}

\author{
Selen Cakmakyapan \\ Department of Statistics, Hacettepe University, \\ Ankara,Turkey \\ selencakmakyapan@hacettepe.edu.tr \\ Gamze Ozel \\ Department of Statistics, Hacettepe University, \\ Ankara,Turkey \\ gamzeozl@hacettepe.edu.tr \\ Yehia Mousa Hussein El Gebaly \\ Department of Statistics, Mathematics and Insurance, Benha University, Egypt \\ yehia1958@hotmail.com \\ G. G. Hamedani \\ Department of Mathematics, Statistics and Computer Science \\ Marquette University, USA \\ gholamhoss.hamedani@marquette.edu \\ Received 1 November 2016 \\ Accepted 20 March 2017
}

\begin{abstract}
In this paper, we define and study a new lifetime model called the Kumaraswamy Marshall-Olkin log-logistic distribution. The new model has the advantage of being capable of modeling various shapes of aging and failure criteria. The new model contains some well-known distributions as special cases such as the Marshall-Olkin loglogistic, log-logistic, lomax, Pareto type II and Burr XII distributions. Some of its mathematical properties including explicit expressions for the quantile and generating functions, ordinary moments, skewness, kurtosis are derived. The maximum likelihood estimators of the unknown parameters are obtained. The importance and flexibility of the new model is proved empirically using a real data set.
\end{abstract}

Keywords: Kumaraswamy-G, Maximum likelihood, Log-Logistic, Order statistic.

2000 Mathematics Subject Classification: 60E05, 62P99

\section{Introduction}

There has been an increased interest in defining new generated classes of univariate continuous distributions by introducing additional shape parameter(s) to a baseline model. The extended distributions have attracted several statisticians to develop new models. The addition of parameters has been proven to be useful in 
exploring skewness and tail properties, and also for improving the goodness-of-fit of the generated family. The well-known generators are the following: the Marshall-Olkin distribution family by Marshall and Olkin (1997), the beta-G by Eugene et al. (2002), the Kumaraswamy-G (Kw-G) by Cordeiro and de Castro (2011), the Logistic-G by Torabi and Montazari (2014), the transformed-transformer (T-X) by Alzaatreh et al. (2013), the odd exponentiated generalized by Cordeiro et al. (2013), the Weibull-G by Bourguignon et al. (2014), the Kumaraswamy Marshal-Olkin distribution family by Alizadeh et al. (2015), the transmuted geometric-G by Afify et al. (2016a) and the beta transmuted-H by Afify et al. (2017).

Marshall and Olkin (1997) proposed a flexible family of distributions and introduced an interesting method of adding a new parameter to an existing distribution. The resulting new distribution includes the original distribution as a special case and gives more flexibility to model various types of data. For further information about the Marshall-Olkin family of distributions, see Barreto-Souza et al. (2013). The log-logistic (LL) distribution (known as the Fisk distribution in economics) has been widely used particularly in survival and reliability over the last few decades. It is the probability distribution of a random variable whose logarithm has a logistic distribution, an alternative to the log-normal distribution since it presents a failure rate function that increases initially and decreases later. The cumulative distribution function (cdf) and probability density function (pdf) of the LL distribution are given (for $x>0$ ) by

$$
G_{L L}(x ; \gamma, \alpha)=1-\left[1+\left(\frac{x}{\alpha}\right)^{\gamma}\right]^{-1} \text { and } g_{L L}(x ; \gamma, \alpha)=\frac{\gamma x^{\gamma-1}}{\alpha^{\gamma}}\left[1+\left(\frac{x}{\alpha}\right)^{\gamma}\right]^{-2},
$$

respectively, where $\alpha>0$ is the scale parameter and $\gamma>0$ is the shape parameter.

Searching a more flexible LL distribution, many authors defined generalizations and modified forms of the LL distribution, with different number of parameters. For example, the Kumaraswamy log-logistic (de Santana et al., 2012), Marshall-Olkin LL (MOLL) (Gui, 2013), Lomax log-logistic (Cordeiro et al., 2014), McDonald log-logistic (Tahir et al., 2014), beta log-logistic (Lemonte, 2014), transmuted log-logistic (Granzotto and Louzada, 2015), Kumaraswamy transmuted log-logistic (Afify et al., 2016b) and generalized transmuted log-logistic (GTLL) (Nofal et al., 2017) distributions.

Gui (2013) defined the cdf and pdf of the MOLL distribution (for $x>0$ ) by

$$
G_{M O L L}(x ; \gamma, \alpha, \beta)=1-\frac{\beta \alpha^{\gamma}}{\left(\mathrm{x}^{\gamma}+\beta \alpha^{\gamma}\right)} \text { and } g_{M O L L}(x ; \gamma, \alpha, \beta)=\frac{\beta \gamma \alpha^{\gamma} x^{\gamma-1}}{\left(\mathrm{x}^{\gamma}+\beta \alpha^{\gamma}\right)^{2}},
$$

respectively, where $\alpha, \gamma, \beta>0$. For $\beta=1$, we obtain the LL distribution.

The goal of this paper is to define and study a new lifetime model called the Kumaraswamy Marshall-Olkin Log-Logistic ("KMOLL" for short) distribution. The main feature of this model is that two additional shape parameters are inserted in (2) to give more flexibility in the form of the generated distribution. Based on the Kumaraswamy-generalized (K-G) family proposed by Cordeiro and de Castro (2011), we construct the new five-parameter KMOLL distribution. We give some mathematical properties of the new distribution with the hope that it will attract wider applications in engineering, reliability, life testing and other research. In fact, the KMOLL distribution can provide better fits than other models.

Let $g(x)$ and $G(x)$ denote the pdf and cdf of the baseline model. Cordeiro and de Castro (2011) defined the cdf of the K-G family by

$$
F(x)=1-\left\{1-G(x)^{a}\right\}^{b} .
$$

The corresponding pdf of (1.3) is given by

$$
f(x)=\operatorname{abg}(x) G(x)^{a-1}\left\{1-G(x)^{a}\right\}^{b-1},
$$


where $a>0$ and $b>0$ are two extra shape parameters whose role are to govern skewness and tail weights. Clearly, for $a=b=1$, we obtain the baseline distribution.

To this end, we start from the MOLL distribution to define the new KMOLL distribution by inserting (1.2) in equations (1.3) and (1.4). Then, the cdf (for $x>0$ ) of the KMOLL distribution is given by

$$
F(x ; a, b, \gamma, \alpha, \beta)=1-\left[1-\left(\frac{\mathrm{x}^{\gamma}}{\mathrm{x}^{\gamma}+\beta \alpha^{\gamma}}\right)^{a}\right]^{b} .
$$

The corresponding pdf of (1.5) is

$$
f(x ; a, b, \gamma, \alpha, \beta)=\frac{a b \beta \gamma \alpha^{\gamma} \mathrm{x}^{\gamma a-1}}{\left(\mathrm{x}^{\gamma}+\beta \alpha^{\gamma}\right)^{a+1}}\left[1-\left(\frac{\mathrm{x}^{\gamma}}{\mathrm{x}^{\gamma}+\beta \alpha^{\gamma}}\right)^{a}\right]^{b-1} .
$$

where $\alpha$ is a scale parameter and the shape parameters $a, b, \gamma$ and $\beta$ govern the skewness of (1.6).

A random variable $X$ with the pdf (1.6) is denoted by $X \sim \operatorname{KMOLL}(a, b, \alpha, \gamma, \beta)$. The survival function, hazard rate function (hrf) and cumulative hazard rate function (chrf) of $X$ are, respectively, given by

$$
\begin{gathered}
\bar{F}(x ; a, b, \gamma, \alpha, \beta)=\left[1-\left(\frac{\mathrm{x}^{\gamma}}{\mathrm{x}^{\gamma}+\beta \alpha^{\gamma}}\right)^{a}\right]^{b}, \\
h(x ; a, b, \gamma, \alpha, \beta)=a b \frac{\beta \gamma \alpha^{\gamma} x^{\gamma-1}}{\left(\mathrm{x}^{\gamma}+\beta \alpha^{\gamma}\right)^{2}}\left(\frac{\mathrm{x}^{\gamma}}{\mathrm{x}^{\gamma}+\beta \alpha^{\gamma}}\right)^{a-1}\left[1-\left(\frac{\mathrm{x}^{\gamma}}{\mathrm{x}^{\gamma}+\beta \alpha^{\gamma}}\right)^{a}\right]^{-1}
\end{gathered}
$$

and

$$
H(x ; a, b, \gamma, \alpha, \beta)=-b \log \left\lfloor 1-\left(\frac{\mathrm{x}^{\gamma}}{\mathrm{x}^{\gamma}+\beta \alpha^{\gamma}}\right)^{a}\right\rfloor .
$$

Some of the possible shapes of the pdf (1.6) for selected parameter values are illustrated in Figure 1. As seen from Figure 1, the density function can take various forms depending on the parameter values. It is evident that the KMOLL distribution is much more flexible than the MOLL distribution, i.e. the additional shape parameters $a$ and $b$ allow for a high degree of flexibility of the KMOLL distribution. Both unimodal and monotonically decreasing shapes appear to be possible. 

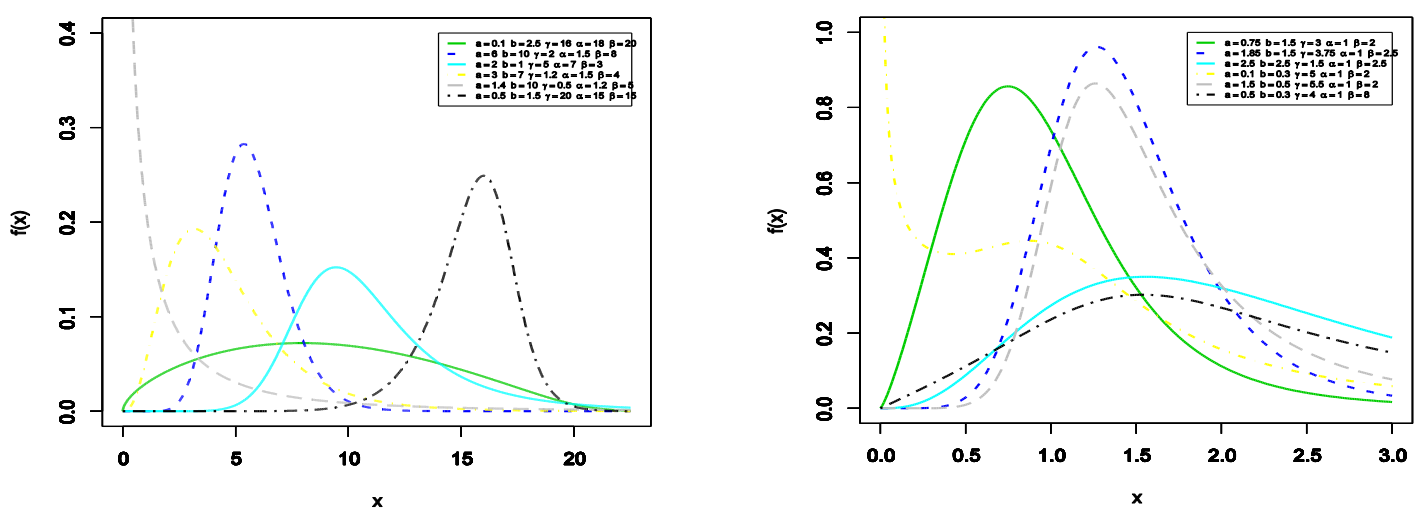

Fig. 1. Plots for the pdf of the KMOLL distribution for several parameter values.

Plots for the hrf of the KMOLL distribution for several parameter values are displayed in Figure 2. Figure 2 shows that the hrf of the KMOLL distribution can be bathtub, upside down bathtub (unimodal), increasing or decreasing. This attractive flexibility makes the hrf of the KMOLL useful and suitable for non-monotone empirical hazard behaviors which are more likely to be encountered or observed in real life situations.
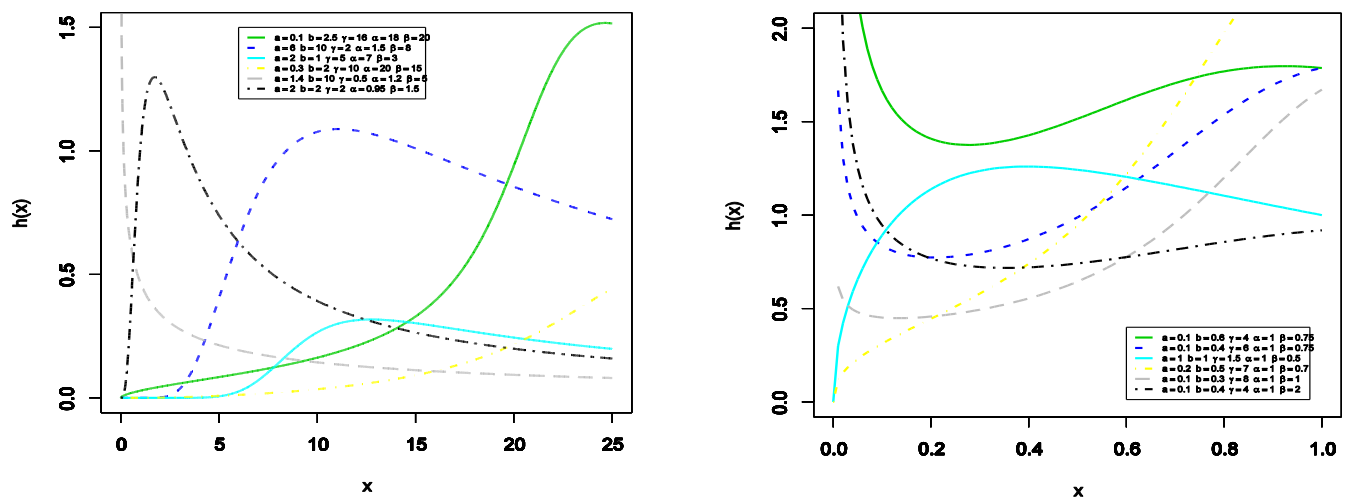

Fig. 2. Plots of the hrf of the KMOLL distribution for several parameter values.

We now state a useful expansion for the KMOLL density. Using the binomial expansion, the pdf of the KMOLL reduces to

$$
f(x)=g(x) \sum_{i=1}^{\infty} w_{i} G^{a(i+1)-1}(x)
$$

where $w_{i}=(-1)^{i} a b\left(\begin{array}{c}b-1 \\ i\end{array}\right)$

The importance of the KMOLL distribution is that it contains as special sub-models several well-known distributions. Table 1 lists the special distributions related to KMOLL distribution. 
Table 1. Sub-models of the KMOLL distribution

\begin{tabular}{llllll}
\hline Reduced Model & $a$ & $b$ & $\gamma$ & $\alpha$ & $\beta$ \\
\hline KLL & $a$ & $b$ & $\gamma$ & $\alpha$ & 1 \\
MOLL & 1 & 1 & $\gamma$ & $\alpha$ & $\beta$ \\
EMOLL & $a$ & 1 & $\gamma$ & $\alpha$ & $\beta$ \\
GMOLL & 1 & $b$ & $\gamma$ & $\alpha$ & $\beta$ \\
ELL & $a$ & 1 & $\gamma$ & $\alpha$ & 1 \\
GLL & 1 & $b$ & $\gamma$ & $\alpha$ & 1 \\
LL & 1 & 1 & $\gamma$ & $\alpha$ & 1 \\
Dagum & $a$ & 1 & $\gamma$ & - & $\alpha \beta^{1 / \gamma}$ \\
BurrXII & 1 & $b$ & 1 & - & $\alpha \beta$ \\
EBurrXII & $a$ & $b$ & 1 & - & $\alpha \beta$ \\
ParetoII & 1 & $1 / b$ & 0 & - & $\alpha \beta / b$ \\
EParetoII & $a$ & $1 / b$ & 0 & - & $\alpha \beta / b$ \\
Lomax & $a$ & $1 / b$ & - & - & $\alpha \beta / b$ \\
\hline
\end{tabular}

The rest of the article is outlined as follows. In Section 2, we obtain the quantile function, shapes, skewness, kurtosis, moments, moment generating functions, Rényi entropies, reliability function and order statistics of $X$. Certain characterizations are presented in Section 3. The maximum likelihood estimates (MLEs) of the model parameters are obtained in Section 4. An application to real data set is considered in Section 5. Finally, Section 6 provides some concluding remarks.

\section{The KMOLL Properties}

In this section, we investigate mathematical properties of the KMOLL distribution including quantile function, skewness, kurtosis, shapes of functions, moments, the Rényi and Shannon entropies, reliability and order statistics.

\subsection{Quantile function}

Quantile functions are in widespread use in statistics and often find representations in terms of lookup tables for key percentiles. Let $X \sim \operatorname{KMOLL}(a, b, \alpha, \gamma, \beta)$. The quantile function say $Q(u)$ is defined by inverting $F(x)$ in (1.5) as

$$
x=Q(u)=\alpha \beta^{1 / \gamma}\left[\left[1-(1-u)^{1 / b}\right]^{-1 / a}-1\right]^{-1 / \gamma} .
$$

The effect of the shape parameters $a, b, \alpha, \gamma, \beta$ on the skewness and kurtosis can be considered based on quantile measures. There are many heavy tailed distributions for which this measure is infinite. So, it becomes uninformative precisely when it needs to be. The Bowley's skewness is based on quartiles:

$$
S=\frac{Q(3 / 4)-2 Q(1 / 2)+Q(1 / 4)}{Q(3 / 4)-Q(1 / 4)}
$$

and the Moors' kurtosis is based on octiles:

$$
K=\frac{Q(7 / 8)-Q(5 / 8)+Q(3 / 8)-Q(1 / 8)}{Q(6 / 8)-Q(2 / 8)}
$$


where $Q($.) represents the quantile function of $X$. These measures are less sensitive to outliers and they exist even for distributions without moments. Skewness measures the degree of the long tail and kurtosis is a measure of the degree of tail heaviness. When the distribution is symmetric, $S=0$ and the when the distribution is right (or left) skewed, $S>0$ or $(S<0)$. As K increases, the tail of the distribution becomes heavier.

\subsection{Moments and moment generating function}

Some of the most important features and characteristics of a distribution can be studied through moments (e.g. tendency, dispersion, skewness and kurtosis). Now we obtain ordinary moments and the moment generating function of the KMOLL distribution. The ordinary moments $E\left(X^{n}\right)=\mu_{n}^{\prime}, n=1,2, \ldots$, of the KMOLL distribution can be obtained, using (1.7), as

$$
\mu_{n}^{\prime}=\sum_{i=0}^{\infty} w_{i} \int_{0}^{\infty} x^{n} g_{\text {MOLL }}(x) G_{\text {MOL }}^{a(i+1)-1}(x) d x,
$$

where $w_{i}=(-1)^{i} a b\left(\begin{array}{c}b-1 \\ i\end{array}\right)$. Here, $g_{\text {MOLL }}($.$) and G_{\text {MOLL }}($.$) are the pdf and cdf of the MOLL distribution,$ respectively. Then, the integral part in (2.1) is defined as

$$
\int_{0}^{\infty} x^{n} g_{\text {MOLL }}(x) G_{\text {MOL }}^{a(i+1)-1}(x) d x=\int_{0}^{1} Q_{\text {MOLL }}^{n}(u) u^{a(i+1)-1} d u,
$$

where $Q_{\text {MOLL }}($.$) is the quantile function of the MOLL distribution for 0<u<1$. Then, we obtain

$$
\mu_{n}^{\prime}=\beta^{n / \gamma} \alpha^{n} \sum_{i=0}^{\infty} w_{i} B\left(\frac{n}{\gamma}+a(i+1), 1-\frac{n}{\gamma}\right),
$$

where $B(.,$.$) is the beta function.$

Further, the central moments $\left(\mu_{n}\right)$ and cumulants $\left(\kappa_{n}\right), n=1,2, \ldots$, of the KMOLL distribution can be obtained from

$$
\mu_{n}=\sum_{k=0}^{n}(-1)^{k}\left(\begin{array}{l}
n \\
k
\end{array}\right) \mu_{1}^{\prime n} \mu_{n-k}^{\prime} \text { and } \kappa_{n}=\mu_{n}^{\prime}-\sum_{k=0}^{n}(-1)^{k}\left(\begin{array}{l}
n \\
k
\end{array}\right) \mu_{1}^{\prime k} \mu_{n-k}^{\prime} .
$$

Here, $\kappa_{1}=\mu_{1}^{\prime}, \kappa_{2}=\mu_{2}^{\prime}-\mu_{1}^{\prime 2}, \kappa_{3}=\mu_{3}^{\prime}-3 \mu_{2}^{\prime} \mu_{1}^{\prime}+2 \mu_{1}^{\prime 3}, \kappa_{4}=\mu_{4}^{\prime}-4 \mu_{3}^{\prime} \mu_{1}^{\prime}-3 \mu_{2}^{\prime 2}+12 \mu_{2}^{\prime} \mu_{1}^{\prime 2}-6 \mu_{1}^{\prime 4}$ etc.

The skewness $\gamma_{1}=\kappa_{3} / \kappa_{2}^{3 / 2}$ and kurtosis $\gamma_{2}=\kappa_{4} / \kappa_{2}^{2}$ are also computed from the second, third and fourth cumulants. Table 2 gives moments, skewness, and kurtosis of the KMOLL distribution for some parameter values.

Table 2 indicates that the skewness value can be positive and negative, also close to zero. Hence, the KMOLL distribution can be right-skewed, left-skewed or symmetric. 
Table 2. Moments, skewness and kurtosis of the KMOLL distribution for some parameter values

\begin{tabular}{lllllll}
\hline KMOLL $(a, b, \gamma, \alpha, \beta)$ & $\mu_{1}^{\prime}$ & $\mu_{2}^{\prime}$ & $\mu_{3}^{\prime}$ & $\mu_{4}^{\prime}$ & $S$ & $K$ \\
\hline$(0.1,1.98,16,18,20)$ & 11.806 & 167.936 & 2656.865 & 45090.060 & 0.000 & -0.811 \\
$(5,10,2,3,8)$ & 11.362 & 133.290 & 1612.749 & 20108.035 & 0.335 & 0.357 \\
$(2,1,5,4,3)$ & 6.228 & 47.418 & 526.448 & 185560.800 & 4.883 & 2403.251 \\
$(4,9,1.2,1.5,8)$ & 10.148 & 119.066 & 1601.209 & 24581.778 & 1.028 & 2.180 \\
$(0.4,15,0.5,1.2,5)$ & 0.018 & 0.002 & 0.000 & 0.000 & 5.511 & 53.883 \\
$(7,2,3.5,3,0.2)$ & 0.565 & 0.356 & 0.258 & 0.230 & 2.200 & 13.764 \\
$(1,4,1,8,1)$ & 0.807 & 0.668 & 0.567 & 0.491 & -0.207 & 0.262 \\
$(0.5,9,16,1,13)$ & 4.126 & 86.379 & 4204.147 & 381961.900 & 5.671 & 63.640 \\
$(1.5,2.5,2,7,4)$ & 4.163 & 17.806 & 78.167 & 352.098 & 0.303 & 0.765 \\
$(25,10,0.5,1.2,1)$ & 3.660 & 15.332 & 73.762 & 409.965 & 1.287 & 3.412 \\
\hline
\end{tabular}

Figure 3 also depicts plots for the skewness and kurtosis coefficients related to additional parameters. In the figure, a parameter decreases while other parameters are kept fixed. These plots indicate that both measures can be very sensitive on these shape parameters. Thus, indicating the importance of the proposed distribution.
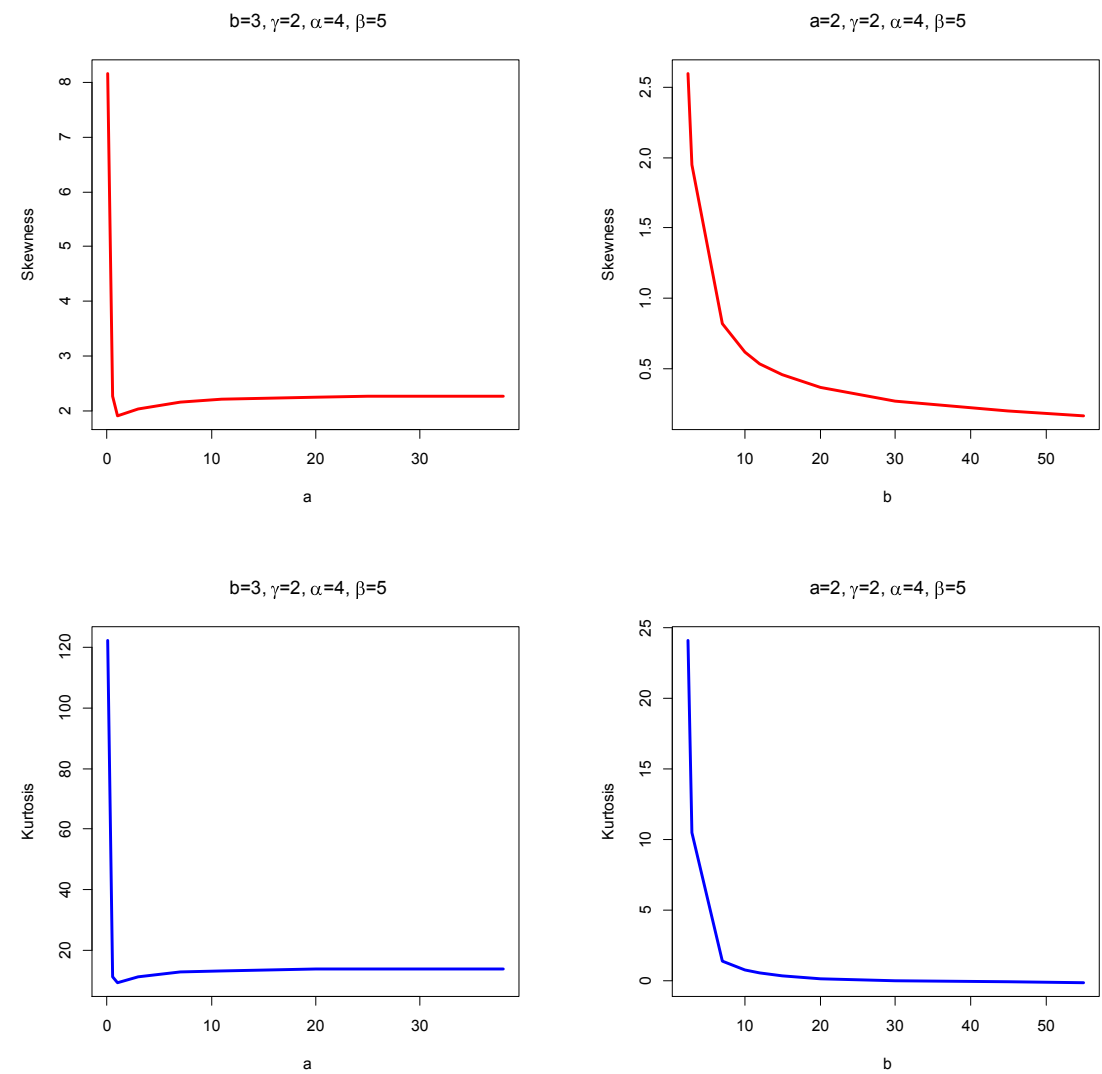

Fig. 3. Skewness and kurtosis plots of the KMOLL distribution for some parameter values

The moment generating function (mgf) is widely used as an alternative way to analytical results compared with working directly with pdf and cdf. The mgf of $X$ is 


$$
M(t)=E\left(e^{t x}\right)=\sum_{n=0}^{\infty} \frac{\mu_{n}^{\prime}}{n !} t^{n},
$$

or another representation for $M(t)$ can be obtained using (1.7)

$$
M(t)=\sum_{i=0}^{\infty} w_{i} \int_{0}^{\infty} e^{t x} g_{\text {MOLL }}(x) G_{\text {MOLL }}^{a(i+1)-1}(x) d x
$$

where $\quad w_{i}=(-1)^{i} a b\left(\begin{array}{c}b-1 \\ i\end{array}\right)$. Then, the integral part in (2.2) is given as $\int_{0}^{\infty} e^{t x} g_{\text {MOLL }}(x) G_{\text {MOLL }}^{a(i+1)-1}(x) d x=\int_{0}^{1} e^{t Q_{\text {MOL }}(u)} u^{a(i+1)-1} d u$ where $Q_{\text {MOLL }}($.$) is the quantile function of the MOLL distribution$ for $0<u<1$. From the Maclaurin expansion, we obtain $e^{t Q_{\text {MOL }}(u)}=\sum_{j=0}^{\infty}\left[t \alpha\left(\frac{\beta u}{1-u}\right)^{1 / \gamma}\right]^{j} / j !$.

Then we obtain

$$
\begin{aligned}
M(t) & =\sum_{j=0}^{\infty} \frac{(t \alpha)^{j} \beta^{j / \gamma}}{j !} \int_{0}^{1} u^{j / \gamma+a(i+1)-1}(1-u)^{-j / \gamma} d u=\sum_{i=0}^{\infty} \sum_{j=0}^{\infty} w_{i} \frac{(t \alpha)^{j} \beta^{j / \gamma}}{j !} B\left(\frac{j}{\gamma}+a(i+1), 1-\frac{j}{\gamma}\right) \\
& =\sum_{j=0}^{\infty} \frac{(t \alpha)^{j} \beta^{j / \gamma}}{j !} B\left(\frac{j}{\gamma}+a(i+1), 1-\frac{j}{\gamma}\right) .
\end{aligned}
$$

\subsection{Unimodality}

The pdf of the KMOLL model is decreasing or unimodal. In order to investigate the critical points of its density function, its first derivative with respect to $\mathrm{x}$ is

$$
\begin{aligned}
\frac{d}{d x} f(x)= & \frac{b(b-1) a^{2} \beta^{2} \gamma^{2} \alpha^{2 \gamma} x^{2 \gamma-2}\left(\frac{x^{\gamma}}{x^{\gamma}+\beta \alpha^{\gamma}}\right)^{2 a-2}\left(-\left(\frac{x^{\gamma}}{x^{\gamma}+\beta \alpha^{\gamma}}\right)^{a}+1\right)^{b-2}}{\left(x^{2 \gamma}+2 \beta(x \alpha)^{\gamma}+\beta^{2} \alpha^{2 \gamma}\right)^{2}} \\
& +\frac{a b(a-1) \beta^{2} \gamma^{2} \alpha^{2 \gamma} x^{2 \gamma-2}\left(\frac{x^{\gamma}}{x^{\gamma}+\beta \alpha^{\gamma}}\right)^{a-2}\left(-\left(\frac{x^{\gamma}}{x^{\gamma}+\beta \alpha^{\gamma}}\right)^{a}+1\right)^{b-1}}{\left(x^{2 \gamma}+2 \beta(x \alpha)^{\gamma}+\beta^{2} \alpha^{2 \gamma}\right)^{2}} \\
& -\frac{2 a b \beta \gamma^{2} \alpha^{\gamma} x^{2 \gamma-2}\left(\frac{x^{\gamma}}{x^{\gamma}+\beta \alpha^{\gamma}}\right)^{a-1}\left(-\left(\frac{x^{\gamma}}{x^{\gamma}+\beta \alpha^{\gamma}}\right)^{a}+1\right)^{b-1}}{\left(x^{\gamma}+\beta \alpha^{\gamma}\right)^{3}} \\
& +\frac{a b \beta \gamma(\gamma-1) \alpha^{\gamma} x^{\gamma-2}\left(\frac{x^{\gamma}}{x^{\gamma}+\beta \alpha^{\gamma}}\right)^{a-1}\left(-\left(\frac{x^{\gamma}}{x^{\gamma}+\beta \alpha^{\gamma}}\right)^{a}+1\right)^{b-1}}{\left(x^{\gamma}+\beta \alpha^{\gamma}\right)^{2}}=0 .
\end{aligned}
$$

There may be more than one root to (2.4). If $\mathrm{x}=\mathrm{x}_{0}$ is a root of (2.4), then it corresponds to a local maximum if $d f(x) / d x>0$ for all $x<x_{0}$ and $d f(x) / d x<0$ for all $x>x_{0}$. It corresponds to a local minimum if 
$d f(x) / d x<0$ for all $x<x_{0}$ and $d f(x) / d x>0$ for all $x>x_{0}$. It corresponds to a point of inflexion if either $d f(x) / d x>0$ for all $x \neq x_{0}$ or $d f(x) / d x<0$ for all $x \neq x_{0}$.

\subsection{Entropies}

The entropy of a random variable $X$ with density function $f(x)$ is a measure of variation of the uncertainty. Two popular entropy measures are the Rényi and Shannon entropies (Rényi (1961). Shannon (1951)). Here. we derive expressions for the Rényi and the Shannon entropies of the KMOLL distribution. The Rényi entropy of a random variable with pdf $f(x)$ is defined as

$$
\mathrm{I}_{R}(\delta)=\frac{1}{1-\delta} \log \int_{-\infty}^{\infty} f^{\delta}(x) d x
$$

for $\delta>0$ and $\delta \neq 1$. Then, we can write

$$
\begin{aligned}
\int_{0}^{\infty} f^{\delta}(x) d x & =\int_{0}^{\infty}\left[g_{\text {MOLL }}(x) \sum_{i=0}^{\infty} w_{i} G_{\text {MOLL }}^{a(i+1)-1}(x)\right]^{\delta} d x=\int_{0}^{\infty} g_{\text {MOLL }}^{\delta}(x) G_{\text {MOLL }}^{(a-1) \delta}(x)\left[\sum_{i=0}^{\infty} w_{i} G_{\text {MOLL }}^{a i}(x)\right]^{\delta} d x \\
& =\sum_{i=0}^{\infty} c_{\delta, i} \int_{0}^{\infty} g_{\text {MOLL }}^{\delta}(x) G_{\text {MOLL }}^{(a-1) \delta+a i}(x) d x,
\end{aligned}
$$

where $w_{i}=(-1)^{i} a b\left(\begin{array}{c}b-1 \\ i\end{array}\right)$ and $\quad c_{\delta, i}=\left(i w_{0}\right)^{-1} \sum_{m=1}^{i}[m(\delta+1)-i] w_{m} c_{\delta, i-m}$.

The integral part of (2.5) is

$$
\begin{aligned}
\int_{0}^{\infty} g_{\text {MOLL }}^{\delta}(x) G_{\text {MOLL }}^{(a-1) \delta+a i}(x) d x & =\int_{0}^{1} u^{a i+\delta a-\delta} g_{\text {MOLL }}^{\delta-1} Q_{\text {MOLL }}(u) d u \\
& =\left(\gamma \alpha^{-1} \beta^{-1 / \gamma}\right)^{\delta-1} \int_{0}^{1} u^{a i+\delta a-\delta+\frac{(\gamma-1)(\delta-1)}{\gamma}}(1-u)^{\frac{(\gamma+1)(\delta-1)}{\gamma}} d u \\
& =\left(\gamma \alpha^{-1} \beta^{-1 / \gamma}\right)^{\delta-1} B\left(a i+\delta a-\delta+\frac{(\gamma-1)(\delta-1)}{\gamma}+1, \frac{(\gamma+1)(\delta-1)}{\gamma}+1\right) .
\end{aligned}
$$

Then the Rényi entropy of the KMOLL distribution is given by

$$
\mathrm{I}_{R}(\delta)=\frac{1}{1-\delta} \log \left\lfloor\left(\gamma \alpha^{-1} \beta^{-1 / \gamma}\right)^{\delta-1} B\left(a i+\delta a-\delta+\frac{(\gamma-1)(\delta-1)}{\gamma}+1, \frac{(\gamma+1)(\delta-1)}{\gamma}+1\right)\right\rfloor .
$$

The Shannon entropy plays a similar role as the kurtosis measure in comparing the shapes of various densities and measuring heaviness of tails. The Shannon entropy of a random variable $\mathrm{X}$ is defined by $E\lfloor-\log f(x)\rfloor$. It is the special case of Rényi entropy when $\delta>1$. The Shannon entropy of the KMOLL distribution is

$$
\begin{aligned}
E[-\log f(x)\rfloor= & -\log (a b)-E\left[\log g_{\text {MOLL }}(x)\right]-(a-1) E\left[\log G_{\text {MOLL }}(x)\right] . \\
& -(b-1) E\left\{\log \left[1-G_{\text {MOLL }}^{a}(x)\right]\right\}
\end{aligned}
$$

To obtain three expectations terms given above. We define and compute

$$
E\left[\log g_{\text {MOLL }}(x)\right]=a b \int_{0}^{\infty} \log \left(g_{\text {MOLL }}(x)\right) g_{\text {MOLL }}(x) G_{\text {MоL }}^{a-1}(x)\left[1-G_{\text {MOLL }}^{a}(x)\right]^{b-1} d x=\sum_{k=0}^{\infty} w_{k} I_{k} .
$$

Here we have 


$$
\begin{aligned}
I_{k} & =\int_{0}^{\infty} \log \left[g_{\text {MOLL }}(x)\right] g_{\text {MOLL }}(x) G_{\text {моL }}^{a(k+1)-1}(x) d x=\int_{0}^{1} \log \left\{g_{\text {мOLL }}\left[Q_{\text {MOLL }}(u)\right]\right\} u^{a(k+1)-1} d u \\
& =\int_{0}^{1} \log \left[\gamma \alpha^{-1} \beta^{-1 / \gamma} u^{\frac{\gamma-1}{\gamma}}(1-u)^{\frac{\gamma+1}{\gamma}}\right] u^{a(k+1)-1} d u \\
& =\frac{1}{a k+a}\left(\log \gamma-\log \alpha-\frac{\log \beta}{\gamma}\right)+\log \frac{1}{2} \sum_{j=1}^{\infty} \frac{(-1)^{j-1} 2^{j}}{j(a k+a+1)}{ }_{1} F_{2}(-j, a k+a, a k+a+1,2)
\end{aligned}
$$

where ${ }_{2} F_{1}$ is the generalized hypergeometric function defined by

$$
{ }_{1} F_{2}(-j, a k+a, a k+a+1,2)=\sum_{k=0}^{\infty} \frac{(a)_{k}(b)_{k}}{(c)_{k}} \frac{x^{k}}{k !}
$$

and $(a)_{k}=a(a+1) \ldots(a+k-1)$ denotes ascending factorial.

Similarly, the following expectations are defined for (12) as $E\left[1-G_{\text {моц }}^{a}(x)\right]=b \int_{0}^{1} \log u u^{b-1} d u=-\frac{1}{b}$ and $E\left[\log G_{M O L L}(x)\right]=\frac{b}{a} \int_{0}^{1} \log u(1-u)^{b-1} d u=\left.\frac{b}{a} \frac{\partial B(\alpha+1, b)}{\partial \alpha}\right|_{\alpha=0}=\frac{-C+\psi(b+1)}{a}$.

Here. $C$ is Euler's constant (Nadarajah et al. 2012).

\subsection{Reliability}

In the context of reliability. the stress-strength model describes the life of a component which has a random strength $X_{1}$ that is subjected to a random stress $X_{2}$. The component fails at the instant that the stress applied to it exceeds the strength. and the component will function satisfactorily whenever $X_{1}>X_{2}$. Hence, $R=\operatorname{Pr}\left(X_{2}<X_{1}\right)$ is a measure of component reliability. Here. we obtain the reliability $R$ when $X_{1} \sim \operatorname{KMOLL}\left(a_{1}, b_{1}, \alpha, \gamma, \beta\right)$ and $X_{2} \sim \operatorname{KMOLL}\left(a_{2}, b_{2}, \alpha, \gamma, \beta\right)$ are independent random variables. Probabilities of this form have many applications especially in engineering concepts.

Let $f_{i}$ and $F_{i}$ denote the pdf and cdf of $X_{i}$ for $i=1,2, \ldots$. Then, the reliability function for the KMOLL distribution is given by

$$
R=\int_{0}^{\infty} f_{1}(x) F_{2}(x) d x
$$

The cdf of $X_{2}$ and the pdf of $X_{1}$ are obtained as

$$
F_{2}(x)=1-\left[1-G_{M O L L}^{a_{2}}(x)\right]^{b_{2}}=1-\sum_{k=0}^{\infty} s_{k} G_{M O L L}^{a_{2} k}(x) \text { and } f_{1}(x)=\sum_{i=0}^{\infty} w_{i} G_{M O L L}^{a_{1}(i+1)-1}(x) g_{M O L L}(x),
$$

After some algebra, we arrive at

$$
R=\sum_{i=0}^{\infty} \frac{w_{i}}{a_{1}(i+1)}-\sum_{i=0}^{\infty} \sum_{k=0}^{\infty} \frac{w_{i} s_{k}}{a_{1}(i+1)+a_{2} k} .
$$

where $w_{i}=(-1)^{i} a b\left(\begin{array}{c}b-1 \\ i\end{array}\right)$ and $s_{k}=(-1)^{k}\left(\begin{array}{c}b_{2} \\ k\end{array}\right)$. 


\subsection{Order statistics}

Order statistics make their appearance in many areas of statistical theory and practice. They enter in the problems of estimation and hypotheses testing in a variety of ways. Therefore, we now discuss some properties of the order statistics for the proposed class of distributions. Let $X_{i: n}$ denote the ith order statistic. Nadarajah et al. (2012) obtained the general results for the Kumaraswamy-G distribution. We use the results about the pdf $f_{i: n}(x)$ of the ith order statistic. Then. we can give the pdf $f_{i: n}(x)$ for a random sample $X_{1}, X_{2}, \ldots, X_{n}$ from the KMOLL distribution. It is well-known that

$$
f_{i: n}(x)=\frac{f(x)}{B(i, n-i+1)} F(x)^{i-1}[1-F(x)]^{n-i}
$$

for $i=1,2, \ldots, n$. Using the binomial expansion in the last equation. We obtain

$$
f_{i: n}(x)=\frac{f(x)}{B(i, n-i+1)} \sum_{j=0}^{n-i}(-1)^{j}\left(\begin{array}{c}
n-i \\
j
\end{array}\right) F^{i+j-1}(x) .
$$

The pdf in (2.7) can also be defined as

$$
f_{i: n}(x)=\sum_{r, k=0}^{\infty} q_{r, k} G_{\text {MOLL }}^{a(k+1)+r-1}(x) g_{\text {MOLL }}(x),
$$

where $q_{r, k}=\sum_{j=0}^{n-i} \frac{(-1)^{j}\left(\begin{array}{c}n-i \\ j\end{array}\right) w_{k} p_{r, i+j-1}}{B(i, n-i+1)}$ and $p_{r, u}(a, b)=\sum_{k=0}^{u}(-1)^{k}\left(\begin{array}{l}u \\ k\end{array}\right) \sum_{m=0}^{\infty} \sum_{l=r}^{\infty}(-1)^{m r+l}\left(\begin{array}{l}k b \\ m\end{array}\right)\left(\begin{array}{c}m a \\ l\end{array}\right)\left(\begin{array}{l}l \\ r\end{array}\right)$.

Several mathematical properties for the KMOLL order statistics (mgf, ordinary moments) can be derived from the mixture form in (2.9). Thus, from (2.9). the sth ordinary moment of $X_{i: n}$ is given by

$$
E\left(X_{i: n}^{s}\right)=\sum_{r, k=0}^{\infty} q_{r, k} \int_{0}^{1} Q_{M O L L}^{s}(u) u^{a(k+1)+r-1} d u=\beta^{s / \gamma} \alpha^{s} B\left(\frac{s}{\gamma}+a k+a+r, \frac{s}{\gamma}+1\right) .
$$

Then the mgf of $\mathrm{X}_{i: n}$ is obtained as

$$
M_{i: n}(t)=\sum_{r, k=0}^{\infty} q_{r, k} \int_{0}^{1} e^{t Q_{\text {моц }}(u)} u^{a} d u=\sum_{r, k=0}^{\infty} \sum_{n=0}^{\infty} q_{r, k} \frac{t^{n} \alpha^{n} \beta^{n / \gamma}}{n !} B\left(\frac{n}{\gamma}+a(k+1)+r, 1-\frac{n}{\gamma}\right),
$$

where $q_{r, k}=\sum_{j=0}^{n-i} \frac{(-1)^{j}\left(\begin{array}{c}n-i \\ j\end{array}\right) w_{k} p_{r, i+j-1}}{B(i, n-i+1)}$ and $B(.,$.$) is the beta function.$

\section{Characterizations}

This section deals with various characterizations of KMOLL distribution. These characterizations are based on: (i) the ratio of two truncated moments; (ii) the hazard function and (iii) certain functions of the random variable. It should be mentioned that for characterization (i) the cdf need not have a closed form. We present our characterizations (i) - (iii) in three subsections.

\subsection{Characterizations based on ratio of two truncated moments}

In this subsection, we present characterizations of KMOLL distribution in terms of a simple relationship between two truncated moments. This characterization result employs a theorem due to (Glänzel. 1987). see 
Theorem 3.1 below. Note that the result holds also when the interval $H$ is not closed. Moreover, as mentioned above. It could be also applied when the cdf $F$ does not have a closed form. As shown in (Glänzel, 1990). This characterization is stable in the sense of weak convergence.

Theorem 3.1. Let $(\Omega, F, \boldsymbol{P}) \Omega$, be a given probability space and let $H=[d, e]$ be an interval for some $d<e$ ( $d=-\infty, e=\infty$ might as well be allowed). Let $X: \Omega \rightarrow H$ be a continuous random variable with the distribution function $\mathrm{F}$ and let $\mathrm{g}$ and $\mathrm{h}$ be two real functions defined on $\mathrm{H}$ such that

$$
E[g(X) \mid X \geq x]=E[h(X) \mid X \geq x] \xi(x), x \in H,
$$

is defined with some real function $\xi$. Assume that $g, h \in C^{1}(H), \xi \in C^{2}(H)$ and $F$ is twice continuously differentiable and strictly monotone function on the set $H$. Finally, assume that the equation $\xi h=g$ has no real solution in the interior of $H$. Then, $F$ is uniquely determined by the functions $g, h$ and $\xi$ particularly

$$
F(x)=\int_{a}^{x} C\left|\frac{\xi^{\prime}(u)}{\xi(u) h(u)-g(u)}\right| \exp (-s(u)) d u,
$$

where the function $s$ is a solution of the differential $s^{\prime}=\frac{\xi h}{\xi h-g}$ and $C$ is the normalization constant. such that $\int_{H} d F=1$

Proposition 3.1. Let $X: \Omega \rightarrow(0, \infty)$ be a continuous random variable and let $h(x)=x^{\gamma(1-\alpha)}\left[1-\left(\frac{x^{\gamma}}{x^{\gamma}+\beta \alpha^{\gamma}}\right)^{\alpha}\right]^{1-b}$ and $g(x)=h(x)\left(x^{\gamma}+\beta \alpha^{\gamma}\right)^{-1}$ for $x>0$. The random variable $X$ has pdf (1.6) if and only if the function $\xi$ defined in Theorem 3.1 has the form

$$
\xi(x)=\frac{\alpha}{\alpha+1}\left(x^{\gamma}+\beta \alpha^{\gamma}\right)^{-1}, x>0 .
$$

Proof. Let $X$ be a random variable with pdf (1.6). Then,

$$
(1-F(x)) E[h(x) \mid X \geq x]=b \beta \alpha^{\gamma}\left(x^{\gamma}+\beta \alpha^{\gamma}\right)^{-a}, x>0,
$$

and

$$
(1-F(x)) E[g(x) \mid X \geq x]=\left(\frac{a}{a+1}\right) b \beta \alpha^{\gamma}\left(x^{\gamma}+\beta \alpha^{\gamma}\right)^{-a-1}, x>0,
$$

and finally

$$
\xi(x) h(x)-g(x)=-\frac{1}{a+1} h(x)\left(x^{\gamma}+\beta \alpha^{\gamma}\right)^{-1}, \text { for } x>0 .
$$

Conversely, if $\xi$ is given as above. Then

$$
s^{\prime}(x)=\frac{\xi^{\prime}(x) h(x)}{\xi(x) h(x)-g(x)}=a \gamma x^{\gamma-1}\left(x^{\gamma}+\beta \alpha^{\gamma}\right)^{-1}, x>0,
$$

and hence

$$
s(x)=\log \left\{\left(x^{\gamma}+\beta \alpha^{\gamma}\right)^{a}\right\}, x>0 .
$$


Now, in view of Theorem 3.1. $X$ has density (1.6).

Corollary 3.1. Let $X: \Omega \rightarrow(0, \infty)$ be a continuous random variable and let $h(x)$ be as in Proposition 3.1. The pdf of $X$ is (6) if and only if there exist functions $g$ and $\xi$ defined in Therorem 3.1 satisfying the differential equation

$$
\frac{\xi^{\prime}(x) h(x)}{\xi(x) h(x)-g(x)}=a \gamma x^{\gamma-1}\left(x^{\gamma}+\beta \alpha^{\gamma}\right)^{-1}, \quad x>0 .
$$

The general solution of the differential equation in corollary 3.1 is

$$
\xi(x)=\left(x^{\gamma}+\beta \alpha^{\gamma}\right)^{a}\left\lfloor-\int a \gamma x^{\gamma-1}\left(x^{\gamma}+\beta \alpha^{\gamma}\right)^{-a-1}(h(x))^{-1} g(x)+D\right\rfloor,
$$

where $D$ is a constant. Note that a set of function satisfying the above differential equation is given in Proposition 3.1 with $D=0$ However, it should be also noted that there are other triplets $(h, g, \xi)$ satisfying the conditions of Theorem 3.1.

Remark 3.1. For $b=1$, (Mendoza et al., 2016). we let $h(x)=g(x)\left[x^{\beta}+\alpha^{\beta}\right]^{-1}$ with $g(x)=x^{-\beta(a-1)}$. Then $\xi(x)=\frac{a+1}{a}\left[x^{\beta}+\alpha^{\beta}\right]$ for $x>0$.

The differential equation and general solution in this case are. respectively.

$$
\frac{\xi^{\prime}(x) h(x)}{\xi(x) h(x)-g(x)}=\beta(a+1)\left\lfloor\frac{x^{\beta-1}}{x^{\beta}+\alpha^{\beta}}\right\rfloor, x>0
$$

and

$$
\xi(x)=\left[x^{\beta}+\alpha^{\beta}\right]^{a}\left[-\int \beta(a+1) x^{\beta-1}\left[x^{\beta}+\alpha^{\beta}\right]^{-(a+1)}(h(x))^{-1} g(x) d x+D\right] .
$$

\subsection{Characterization based on hazard function}

It is known that the hazard function. $h_{F}$. a twice differentiable distribution function, $F$, satisfies the first order differential equation

$$
\frac{f^{\prime}(x)}{f(x)}=\frac{h_{F}^{\prime}(x)}{h_{F}(x)}-h_{F}(x) .
$$

For many univariate continuous distributions. this is the only characterization available in terms of the hazard function. The following characterization establishes a characterization of KMOLL distribution which is not of the above trivial form.

Proposition 3.2. Let $X: \Omega \rightarrow(0, \infty)$ be a continuous random variable. The pdf of $\mathrm{X}$ is (1.6) if and only if its hazard function $h_{F}(x)$ satisfies the differential equation

$$
h_{F}^{\prime}(x)-(a \gamma-1) x^{-1} h_{F}(x)=a b \beta \gamma \alpha^{\gamma} x^{a \gamma-1}\left\{\frac{\gamma x^{\gamma-1}\left(x^{\gamma}+\beta \alpha^{\gamma}\right)^{-2}\left[a \beta \alpha^{\gamma} x^{\gamma(a-1)}-(a+1)\left(x^{\gamma}+\beta \alpha^{\gamma}\right)^{a}\right]}{\left[\left(x^{\gamma}+\beta \alpha^{\gamma}\right)^{a}-x^{a \gamma}\right]}\right\},
$$

$x>0$. with the initial condition $h_{F}(0)=0$ for $a \gamma>1$. 
Proof. If $X$ has pdf (1.6) then clearly the above differential equation holds. Now, if it holds, then

$$
\frac{d}{d x}\left\{x^{1-a \gamma} h_{F}(x)\right\}=a b \beta \gamma \alpha^{\gamma} \frac{d}{d x}\left\{\left(x^{\gamma}+\beta \alpha^{\gamma}\right)^{-(a+1)}\left[1-\left(\frac{x^{\gamma}}{x^{\gamma}+\beta \alpha^{\gamma}}\right)^{a}\right]^{-1}\right\},
$$

or

$$
h_{F}(x)=\frac{a b \beta \gamma \alpha^{\gamma} x^{a \gamma-1}\left(x^{\gamma}+\beta \alpha^{\gamma}\right)^{-(a+1)}}{1-\left(\frac{x^{\gamma}}{x^{\gamma}+\beta \alpha^{\gamma}}\right)^{a}}
$$

which is the hazard function of the KMOLL distribution.

Remark 3.2. For $a=b=1$ (special case of (1.4)). we have the following simple differential equation

$$
h_{F}^{\prime}(x)-(\beta-1) x^{-1} h_{F}(x)=-\frac{\beta^{2} x^{2(\beta-1)}}{\left[x^{\beta}+\alpha^{\beta}\right]^{2}}, x>0 .
$$

\subsection{Characterization based on certain functions of the random variable}

The following propositions have already appeared in (Hamedani, 2013). so we will just state them here which can be used to characterize KMOLL distribution.

Proposition 3.3. Let $X: \Omega \rightarrow(d, e)$ be a continuous random variable with cdf $\mathrm{F}$. Let $\psi(x)$ be a differentiable function on $(d, e)$ with $\lim _{x \rightarrow d^{+}} \psi(x)=1$. Then for $\delta \neq 1$.

$$
E[\psi(x) \mid X \geq x]=\delta \psi(x), \quad x \in(d, e),
$$

If and only if

$$
\psi(x)=(1-F(x))^{\frac{1}{\delta}-1}, \quad x \in(d, e)
$$

Remark 3.3. It is easy to see that for certain functions. e.g., $\psi(x)=1-\left(\frac{x^{\gamma}}{x^{\gamma}+\beta \alpha^{\gamma}}\right)^{a}, \delta=\frac{b}{b+1}$ and $(d, e)=(0, \infty)$.

Proposition 3.3 provides a characterization of KMOLL distribution. Clearly there are other suitable functions $\psi$. We chose the above one for simplicity.

\section{Maximum Likelihood Estimation}

Several approaches for parameter estimation have been proposed in the literature but the maximum likelihood method is the most commonly employed. Here we consider estimation of the unknown parameters of the KMOLL distribution by the method of maximum likelihood. Let $x_{1}, x_{2}, \ldots, x_{n}$ be observed values from the KMOLL distribution with parameters $a, b, \gamma, \alpha$ and $\beta$. The log-likelihood function for $(a, b, \gamma, \alpha, \beta)$ is given by $\log L=\log a+\log b+\log \beta+\log \gamma+\gamma \log \alpha+(\gamma a-1) \sum_{i=1}^{n} \log x_{i}-(a+1) \sum_{i=1}^{n} \log \left(x_{i}^{\gamma}+\beta \alpha^{\gamma}\right)+(b-1) \log \left(1-s_{i}{ }^{a}\right)$, 
where $s_{i}=x_{i}^{\gamma} /\left(x_{i}^{\gamma}+\beta \alpha^{\gamma}\right)$.

The derivatives of the log-likelihood function with respect to the parameters $a, b, \gamma, \alpha$ and $\beta$ are given by respectively.

$$
\begin{gathered}
\frac{\partial \log L}{\partial a}=\frac{1}{a}+\gamma \sum_{i=1}^{n} \log x_{i}-\sum_{i=1}^{n} \log \left(x_{i}^{\gamma}+\beta \alpha^{\gamma}\right)-(b-1) \sum_{i=1}^{n}\left(1-s_{i}^{a}\right)^{-1} s_{i}^{a} \log \left(s_{i}\right), \\
\frac{\partial \log L}{\partial b}=\frac{1}{b}+\sum_{i=1}^{n} \log \left(1-s_{i}^{a}\right), \\
\frac{\partial \log L}{\partial \gamma}=\frac{1}{\gamma}+\log \alpha+a \sum_{\mathrm{i}=1}^{\mathrm{n}} \log \mathrm{x}_{\mathrm{i}}-a(b-1) \sum_{i=1}^{n} \frac{\beta \log x_{i}\left(x_{i} \alpha\right)^{\gamma}-\beta \log \alpha\left(x_{i} \alpha\right)^{\gamma}}{\left(x_{i}^{2 \gamma}+2 \beta\left(x_{i} \alpha\right)^{\gamma}+\beta^{2} \alpha^{2 \gamma}\right)\left(1-s_{i}^{a}\right)}+a \sum_{i=1}^{n}\left[\log x_{i}-\frac{\beta \alpha^{\gamma} \log \alpha+x_{i}^{\gamma} \log x_{i}}{x_{i}^{\gamma}+\beta \alpha^{\gamma}}\right] \\
-\sum_{i=1}^{n} \frac{\left(\beta \alpha^{\gamma} \log \alpha+x_{i}^{\gamma} \log x_{i}\right)}{x_{i}^{\gamma}+\beta \alpha^{\gamma}}, \\
\frac{\partial \log L}{\partial \alpha}=a \beta \gamma(b-1) \alpha^{\gamma-1} \sum_{i=1}^{n} x_{i}^{\gamma} s_{i}^{a-1}\left(1-s_{i}^{a}\right)^{-1}\left(x_{i}^{\gamma}+\beta \alpha^{\gamma}\right)^{-2}-\left[\beta \gamma(a-1) \alpha^{\gamma-1}+2 \beta \gamma \alpha^{\gamma-1}\right] \sum_{i=1}^{n}\left(x_{i}^{\gamma}+\beta \alpha^{\gamma}\right)^{-1}+\frac{\gamma}{\alpha}, \\
\frac{\partial \log L}{\partial \beta}=\frac{1}{\beta}+a(b-1) \sum_{i=1}^{n} \frac{\left(x_{i} \alpha\right)^{\gamma} s_{i}^{a-1}}{\left(x_{i}^{\gamma}+\beta \alpha^{\gamma}\right)^{2}\left(1-s_{i}^{a}\right)}-(a-1) \alpha^{\gamma}-2 \alpha^{\gamma} \sum_{i=1}^{n}\left(x_{i}^{\gamma}+\beta \alpha^{\gamma}\right)^{-1} .
\end{gathered}
$$

The MLEs of $(\mathrm{a}, \mathrm{b}, \gamma, \alpha, \beta)$, say $(\hat{\mathrm{a}}, \hat{\mathrm{b}}, \hat{\gamma}, \hat{\alpha}, \hat{\beta})$, are the simultaneous solutions of the equations $\frac{\partial \log L}{\partial a}=0$, $\frac{\partial \log L}{\partial b}=0, \frac{\partial \log L}{\partial \gamma}=0, \frac{\partial \log L}{\partial \alpha}=0$ and $\frac{\partial \log L}{\partial \beta}=0$. Maximization of the likelihood function can be performed by using $n l m$ or optimize in R statistical package.

\section{An Illustrative Application}

In this section, we use a real data set to compare the fits of the KMOLL distribution with MOLL, LL and Weibull Fréchet (WFr) (Afify et al., 2016c) distributions. We will use a data set consists of 63 observations of the strengths of $1.5 \mathrm{~cm}$ glass fibres (Smith and Naylor, 1987), originally obtained by workers at the UK National Physical Laboratory. Unfortunately, the measurement units are not given in their paper. We estimate the unknown parameters of the distributions by the maximum likelihood. Then, we provide the values of the following statistics: Akaike Information Criterion (AIC), Consistent Akaike Information Criterion (CAIC) and Bayesian Information Criterion (BIC).

In general, the smaller the values of these statistics, the better the fit to the data. Table 3 lists the MLEs of the parameters and the values of AIC, CAIC and BIC statistics. 
Table 3. MLEs and the values of AIC, CAIC and BIC statistics

\begin{tabular}{|c|c|c|c|c|c|c|c|c|}
\hline Distribution & Estimat & ed Param & eters (StaI & dard Err & & AIC & CAIC & BIC \\
\hline $\operatorname{KMOLL}(a, b, \gamma, \alpha, \beta)$ & $\begin{array}{l}1.8355 \\
(0.096)\end{array}$ & $\begin{array}{l}0.0028 \\
(0.002)\end{array}$ & $\begin{array}{l}47.4236 \\
(13.307)\end{array}$ & $\begin{array}{l}0.0588 \\
(0.030)\end{array}$ & $\begin{array}{l}0.2786 \\
(0.095) \\
\end{array}$ & 28.0861 & 29.1387 & 38.8018 \\
\hline $\operatorname{MOLL}(\gamma, \alpha, \beta)$ & $\begin{array}{l}2.3267 \\
(1.289)\end{array}$ & $\begin{array}{l}0.0353 \\
(0.154)\end{array}$ & $\begin{array}{l}7.9260 \\
(0.873)\end{array}$ & & & 51.5799 & 51.9867 & 58.0093 \\
\hline $\operatorname{LL}(\gamma, \alpha)$ & $\begin{array}{l}1.5262 \\
(0.041) \\
\end{array}$ & $\begin{array}{l}7.9260 \\
(0.873) \\
\end{array}$ & & & & 49.5799 & 49.7799 & 53.8662 \\
\hline $\operatorname{WFr}(\alpha, \beta, a, b)$ & $\begin{array}{l}0.3865 \\
(0.799) \\
\end{array}$ & $\begin{array}{l}0.2436 \\
(0.285) \\
\end{array}$ & $\begin{array}{l}1.4762 \\
(4.782) \\
\end{array}$ & $\begin{array}{l}16.8561 \\
(20.485) \\
\end{array}$ & & 39.0 & 47.6 & 42.4 \\
\hline
\end{tabular}

Table 4. Cramer-von Misses and Anderson Darling statistics

\begin{tabular}{lll}
\hline & A & W \\
\hline $\operatorname{KMOLL}(a, b, \gamma, \alpha, \beta)$ & 0.0181403 & 0.127219 \\
$\operatorname{MOLL}(\gamma, \alpha, \beta)$ & 0.4969404 & 2.748973 \\
$\operatorname{LL}(\gamma, \alpha)$ & 0.4969402 & 2.748972 \\
\hline
\end{tabular}

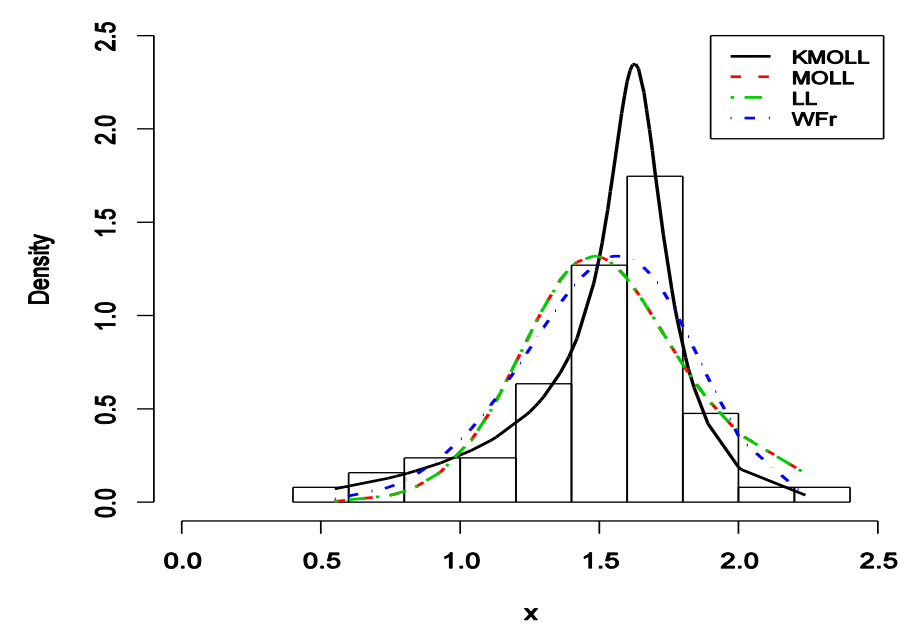

Fig. 4. Fitted densities of the KMOLL, MOLL, LL and WFr distributions for the data set.

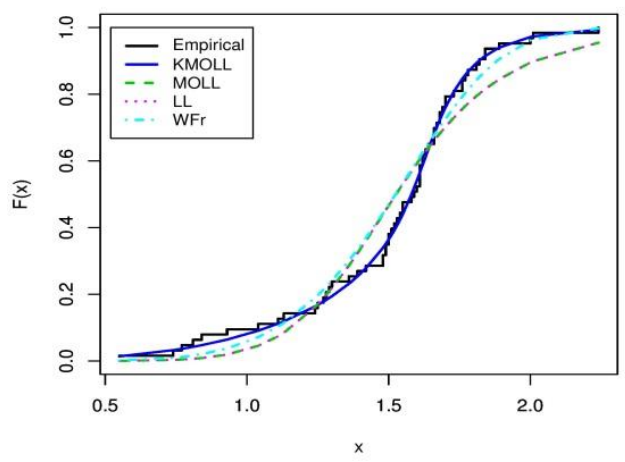

Fig. 5. Estimated and KMOLL, MOLL, LL and WFr cdfs for the data set. 
Based on Table 3, it is clear that KMOLL distribution provides the overall best fit and therefore could be chosen as the more adequate model than other models for explaining the data set. Table 4 gives Cramer-von Misses (W) and Anderson Darling statistics (A) for the three models which are the KMOLL, MOLL and LL distributions. More information is provided by a histogram of the data given in Figure 4. Fitted lines in Figure 4 represent the KMOLL, MOLL, LL and WFr distributions. Figure 5 shows empirical cdf and the fitted cdfs. Finally, we give Q-Q plots for all fitted models. The figures also reveals that the KMOLL fits the data very well.

KMOLL

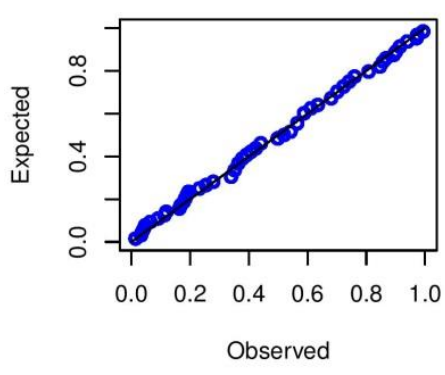

LL

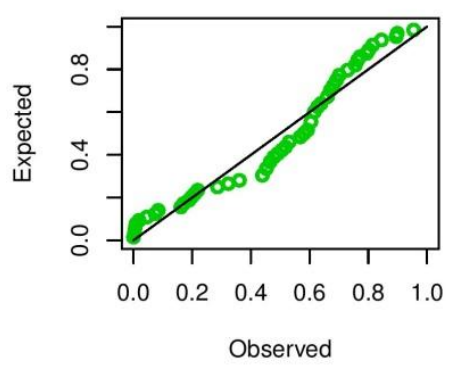

MOLL

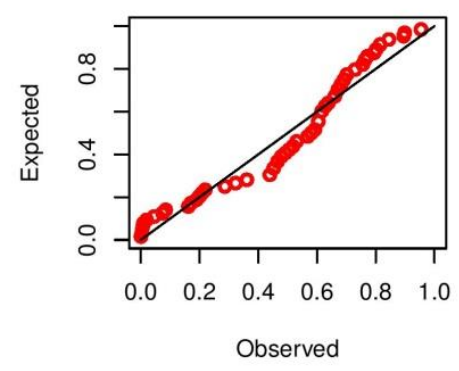

WFr

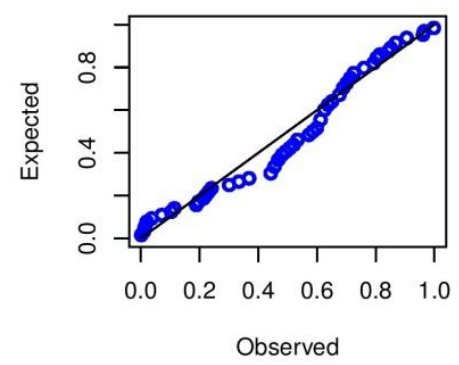

Fig. 6. Q-Q plots for KMOLL, MOLL, LL and WFr distributions.

\section{Conclusion}

In this paper. we introduce a five-parameter distribution called the Kumaraswamy Marshal-Olkin log-logistic (KMOLL) distribution. Interestingly. our proposed model has increasing. upside-down bathtub and bathtub shaped hazard rate function. A study on the mathematical properties of the new distribution is presented. We obtain the moment generating function. ordinary moments. skewness. kurtosis. hazard and survival functions. The estimation of the model parameters is done via maximum likelihood method. We also provide a numerical example of our findings. We hope that the proposed model may attract applications in survival analysis and customer lifetime duration etc.

\section{References}

[1] A. Z. Afify, M. Alizadeh H. M. Yousof.. G. Aryal and M. Ahmad, The transmuted geometric-G family of distributions: theory and applications, Pak. J. Statist. 32 (2016a) 139-160.

[2] Afify A.Z, G. M. Cordeiro, H. M. Yousof, A. Alzaatreh and Z. M. Nofal, The Kumaraswamy transmuted-G family of distributions: Properties and Applications, J. Data Sci. 14(2) (2016b) 245-270. 
[3] A. Z. Afify, M. Haitham, H. M. Yousof, G. M.Cordeiro, E. M. M. Ortega and Z. M. Nofal, The Weibull Fréchet distribution and its applications, J. Appl. Stat. 43(14) (2016c) 2608-2626.

[4] A. Z. Afify, H. M. Yousof and S. Nadarajah, The beta transmuted-H family for lifetime data, Stat. Interface, 10(3) (2017) 505520 .

[5] A. Akinsete, F. Famoye and C. Lee, The beta Pareto distribution, Statistics 42 (2008) 547-563.

[6] M. Alizadeh, M. H. Tahir, G. M. Cordeiro, M. Mansoor, M. Zubair and G.G. Hamedani, The Kumaraswamy Marshal-Olkin family of distributions, J. Egyptian Math. Soc. (2015). http://dx.doi.org/10.1016/j.joems.2014.12.002

[7] A. Alzaatreh, C. Lee and F. Famoye, A new method for generating families of continuous distributions, Metron 71 (2013) 63-79.

[8] M. Bourguignon, R. B. Silva and G. M.Cordeiro, The Weibull-G family of probability distributions, J. Data Sci. 12 (2014) 53-68.

[9] G. M. Cordeiro and M. Castro, A new family of generalized distribution, J. Stat. Comput. Simulat. 81(7) (2011) 883-898.

[10] G. M. Cordeiro, E. M. M. Ortega, B. V. Popovic and R. R. Pescim, The Lomax generator of distributions: properties. minification process and regression model, Appl. Math. Comput. 247 (2014) 465-486.

[11] T. V. F. de Santana., E. M. M. Ortega, G. M. Cordeiro and G. O. Silva, The Kumaraswamy-log-logistic distribution, J. Stat. Theory Appl. 11(3) (2012) 265-291.

[12] N. Eugene, C. Lee and F. Famoye, The beta-normal distribution and its applications. Comm. Stat. Theor. Meth. 31(4) (2002) 497512.

[13] W. Glänzel, A characterization theorem based on truncated moments and its application to some distribution families, Mathematic Statistics and Probability Theory (Bad Tatzmannsdorf. 1986). Vol. B. Reidel. Dordrecht. 1987. 75-84.

[14] W. Glänzel, Some consequences of a characterization theorem based on truncated moments, Statistics: J. Appl. Stat. 21(4) (1990) 613-618.

[15] . D. C. T. Granzotto and F. Louzada, The transmuted log-logistic distribution: modeling. inference and an application to a polled tabapuarace time up to first calving data, Comm. Stat. Theor. Meth. 44 (2015) 3387-3402.

[16] W. Gui, Marshall-Olkin extended log-logistic distribution and its application in minification processes, Appl. Math. Sci. 7 (2013) 3947-396.

[17] G.G. Hamedani, On certain generalized gamma convolution distributions II, Technical Report No. 484, SCS, Arquette University, 2013.

[18] A. J. Lemonte, The beta log-logistic distribution, Braz. J. Prob. Stat. 28 (2014) 313-332.

[19] A. W.Marshall and I. Olkin, A new method for adding a parameter to a family of distributions with application to the exponential and Weibull families, Biometrika 84 (1997) 641-652.

[20] N. V. R. Mendoza, E. M. M. Ortega and G. M.Cordeiro, The exponentiated- log-logistic geometric distribution: dual activation, Commun. Statist. Theo- Meth. 45 (2016). 3838-3859.

[21] S. Nadarajah, G. M.Cordeiro and E. M. M. Ortega, General results for the Kumaraswamy-G distribution, J. Stat. Comput. Simulat. 82 (2012) 951-979.

[22] Z. M. Nofal, A. Z. Afify, H. M. Yousof and G. M. Cordeiro, The generalized transmuted-G family of distributions, Comm. Stat. Theor. Meth. 46(8) (2017) 4119-4136.

[23] A. Rényi, On measures of entropy and information, In: Proceedings of the 4th Berkeley Symposium on Mathematical Statistics and Probability. University of California Press. pp. 547-561. Berkeley (1961)

[24] C.E. Shannon, Prediction and entropy of printed English. Bell Syst. Tech. J. 30 (1951) 50-64.

[25] R. L. Smith and J. C. Naylor, A comparison of maximum likelihood and Bayesian estimators for the three-parameterWeibull distribution, Appl. Statist. 36(1987) (2014) 358-369

[26] M. H. Tahir, M. Mansoor, M. Zubair and G. Hamedani, McDonald log-logistic distribution with an application to breast cancer data, J. Stat. Theory. Pract. 13 (2014) 65-82.

[27] H. Torabi and N. H. Montazari, The logistic-uniform distribution and its application, Commun. Stat. Simulat. Comput. 43 (2014) 2551-2569. 